MIDPI

sciforum
MOL2NET, International Conference Series on Multidisciplinary Sciences

Conference on Molecular, Biomedical, and Computational Sciences and Engineering, 7 th ed.

\title{
Citral, a valuable aid against metabolic inflammation?
}

Vinícius Peixoto Rodrigues ${ }^{a}$, Maycon Tavares Emílio-Silva ${ }^{a}$, Rie Ohara ${ }^{a}$, Gabriela Bueno $^{a}$, Priscila Romano Raimundo ${ }^{a}$, Victoria Gabrielle Gomes ${ }^{a}$, Lúcia Regina Machado da Rocha ${ }^{a}$, Clélia Akiko Hiruma-Lima ${ }^{a}$.

${ }^{a}$ Department of Structural and Functional Biology (Physiology), Institute of Biosciences, São Paulo State University, Botucatu 18618970, São Paulo, Brazil.

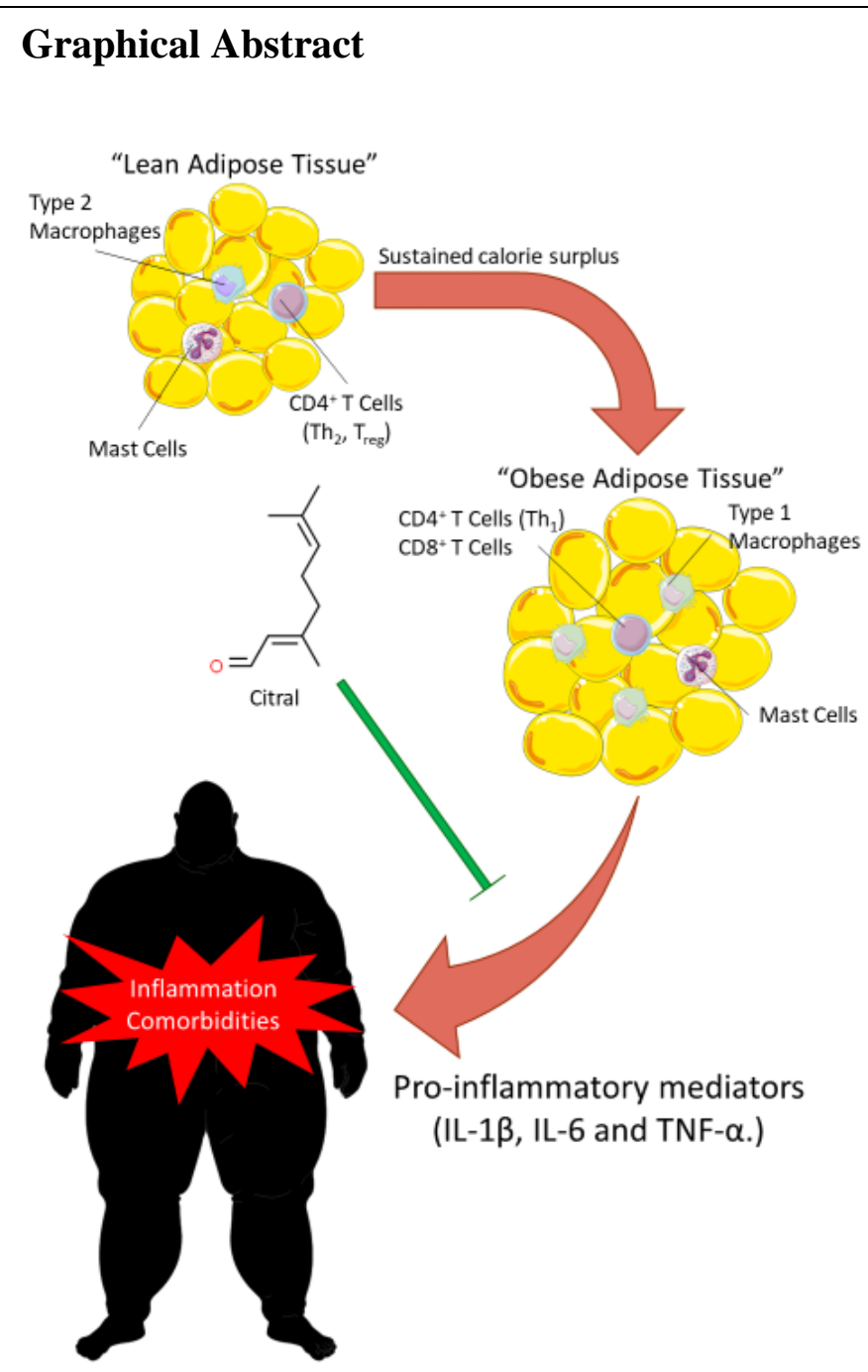

\section{Abstract.}

The adipose tissue (AT) is directly involved in the initiation and maintenance of the inflammatory response responsible for the development of several diseases related to obesity. Citral is a monoterpene found in plants with antiinflammatory and anti-adipogenic potential. The aim of this study was to evaluate the curative effect of citral on pro-inflammatory mediators related to obesity-induced hypercaloric diet (HFD) in experimental animals. Obese Male C57BL/6J mice received Citral treatments orally by gavage $(25,100$ and $300 \mathrm{mg} / \mathrm{kg}$ ) for ten, 17 or 24 days. an oral glucose tolerance test and an insulin tolerance test were performed, and AT stores were collected for inflammatory mediators quantification. All animals fed with HFD showed high fasting basal glycemia when compared to control animals. All animals treated with Citral $(25,100$ and $300 \mathrm{mg} / \mathrm{kg})$ for ten days presented high levels of IL-4, IL-5 and IL-17. After 17 days of treatment, Citral $100 \mathrm{mg} / \mathrm{kg}$ decreased TNF- $\alpha$, IL-6 and IL-1 $\beta$. In addition, Citral was able to increase the concentrations of IL-4 and IL-17. Citral was able to decrease blood glucose without changing insulin sensitivity. A significant decrease in pro-inflammatory and an increase in anti-inflammatory cytokines were observed in HFD-fed animals, indicating an action of Citral in combating metabolic inflammation. 


\section{Introduction}

Obesity is one of the biggest public health problems in the world, the WHO estimates that more than 700 million adults will be obese by 2025 [1]. Obesity is characterized by an excessive accumulation of fat that can compromise health. Adipose tissue (AT) is directly involved in the initiation and maintenance of the inflammatory response triggered by obesity [2]. This metabolic inflammation is responsible for the development of diseases such as type 2 diabetes, cardiovascular diseases and non-alcoholic fatty liver disease [3]. Finding an adequate treatment for obesity and its consequences has proved to be a great challenge. Previous studies have demonstrated the anti-inflammatory and anti-adipogenic potential of Citral, a monoterpene found in plants such as lemon grass and ginger. Citral is an excellent candidate as a therapeutic agent to combat obesity and metabolic inflammation. The aim of this study was to evaluate the curative effect of citral on pro-inflammatory mediators related to obesity-induced hypercaloric diet (HFD) in experimental animals.

\section{Materials and Methods}

Male C57BL/6J mice were subjected to HFD (60\% of calories from lipids) for 12 weeks [4] then received Citral treatments orally by gavage $(25,100$ and $300 \mathrm{mg} / \mathrm{kg}$ ) for ten, 17 and 24 days. To assess the onset of obesity an oral glucose tolerance test [5] and an insulin tolerance test [6] were performed before and after treatments. At the end of each treatment period, adipose tissue stores were collected for quantification of inflammatory mediators by Luminex multiplex assay according to manufacturer instructions (Kit MCYTOMAG-70K; Merck-Millipore) Concomitantly, a group fed with HFD and standard diet (SD, $15 \%$ of calories from lipids) treated with vehicle (Tween 80 at $1 \%, 10 \mathrm{~mL} / \mathrm{kg}$ ) were used as negative and positive controls, respectively. All animal handling and experimental protocols were previously approved by the local ethics committee (protocol $n^{\circ}$ CEUA 1115). Data are presented as mean \pm standard error of the mean, one-way ANOVA with Tukey's post-hoc test and Student's $t$ test were used $(p<0.05)$.

\section{Results and Discussion}

All animals fed with HFD showed high fasting basal glycemia when compared to control animals. All animals treated with Citral $(25,100$ and $300 \mathrm{mg} / \mathrm{kg})$ for ten days presented high levels of IL-4 $(p<0.05)$, IL-5 $(p<0.05)$ and IL-17 $(p<0.01)$ when compared to SD-fed animals. After 17 days of treatment, Citral $100 \mathrm{mg} / \mathrm{kg}$ decreased TNF- $\alpha(p<0.05)$ and IL-6 $(p<0.01)$ levels in relation to the vehicle group and IL$1 \beta$ levels in the Citral group reduced to the level of the SD-fed control group $(p>0.05)$. In addition, Citral $(100 \mathrm{mg} / \mathrm{kg}, 17$-day treatment) was able to increase the concentrations of IL-4 and IL-17 $(p<0.05)$ when compared to animals fed with SD.

\section{Conclusions}

Citral was able to decrease blood glucose without changing insulin sensitivity. A significant decrease in pro-inflammatory mediators and an increase in anti-inflammatory cytokine levels was observed in HFD animals, indicating an action of this monoterpene in combating metabolic inflammation.

\section{References}

1. WHO, W.H.O. Obesity and Overweight Available online: http://www.who.int/news-room/factsheets/detail/obesity-and-overweight.

2. Skurk, T.; Alberti-Huber, C.; Herder, C.; Hauner, H. Relationship between Adipocyte Size and Adipokine Expression and Secretion. The Journal of Clinical Endocrinology \& Metabolism 2007, 92, 1023-1033, doi:10.1210/jc.2006-1055. 
3. Reilly, S.M.; Saltiel, A.R. Adapting to Obesity with Adipose Tissue Inflammation. Nature Reviews Endocrinology 2017, 13, 633-643, doi:10.1038/nrendo.2017.90.

4. de Fraia Pinto, L.; Compri, C.M.; Fornari, J.V.; Bartchewsky, W.; Cintra, D.E.; Trevisan, M.; de Oliveira Carvalho, P.; Ribeiro, M.L.; Velloso, L.A.; Saad, M.J.; et al. The Immunosuppressant Drug, Thalidomide, Improves Hepatic Alterations Induced by a High-Fat Diet in Mice. Liver International 2010, 30, 603-610, doi:10.1111/j.1478-3231.2009.02200.x.

5. Bonora, E.; Targher, G.; Alberiche, M.; Bonadonna, R.C.; Saggiani, F.; Zenere, M.B.; Monauni, T.; Muggeo, M. Homeostasis Model Assessment Closely Mirrors the Glucose Clamp Technique in the Assessment of Insulin Sensitivity: Studies in Subjects with Various Degrees of Glucose Tolerance and Insulin Sensitivity. Diabetes Care 2000, 23, 57-63, doi:10.2337/diacare.23.1.57.

6. Wang, C.-Y.; Liao, J.K. A Mouse Model of Diet-Induced Obesity and Insulin Resistance. Methods in molecular biology (Clifton, N.J.) 2012, 821, 421-433, doi:10.1007/978-1-61779-4308_27. 